\title{
Numerical Simulation of Cavitation of Water Jet Nozzle Based on Realizable $k-\varepsilon$ Model
}

\author{
Yongqiang SU*, Jinfa SHI**, Yahui WANG*** \\ *North China University of Water Resources and Electric Power, Zhengzhou, 460046, China. \\ E-mail: suyongqiang0104@163.com \\ **North China University of Water Resources and Electric Power, Zhengzhou, 460046, China. \\ E-mail: shijinfa@ncwu.edu.cn (Corresponding author) \\ ***North China University of Water Resources and Electric Power, Zhengzhou, 460046, China. \\ E-mail: wangyahui@ncwu.deu.cn \\ crossref http://dx.doi.org/10.5755/j02.mech.28583
}

\section{Introduction}

The nozzle is the actuator of the high pressure water jet generating device, which plays a dominant role in the quality of the resulting water jet and also limits the other components of the system. Therefore, many experts and scholars have conducted a lot of research on the structural parameters of the nozzle. The violent collapse of cavitation bubbles at the boundary of the object produces high-speed micro-jets and high-voltage pulses, which are widely considered to be the cause of material erosion. Therefore, the size of the vapor fraction has a very important influence on the quality of cavitation cleaning. Since the cavitation phenomenon involves complex physical factors such as turbulence and phase transition, the research on cavitation nozzles is mainly based on numerical simulation [1-3]. Reference [4] only qualitatively illustrates that increasing the length-diameter ratio of the nozzle throat is equivalent to increasing the low pressure area. No experiments were conducted to further analyze the simulation results. Liu Wenchuan et al. [5-6] studied the turbulence numerical simulation of fluid passing through a $120^{\circ}$-impinging edge Helmholtz nozzle. The interaction between cavitation and eddy current formation is analyzed by using vorticity transport equation. The advantage is that range of the oscillation frequency, as well as the periodic variation of the cavitation clouds and the vortex structure, was obtained. Huang Biao et al. [7] used het standard RNG $\mathrm{k}-\varepsilon$ based filter model, the modified RNG $\mathrm{k}-\varepsilon$ model, and the FBM model based on modified RNG $k-\varepsilon$ to simulate the Clark-y airfoil cavitation flow, and studied the cloud cavitation phenomena. The advantage is that adopting FBM model based on modified RNG $\mathrm{k}-\varepsilon$ can more accurately simulate the cloud cavitation form and the details of cavitation shedding. Anirban Guha et al. [8] studied the water jet cleaning process through experiments and numerical simulation, and deduced a theoretical model for evaluating the optimal and critical interval distance. The advantage is that the proposed simulation methodology can be helpful for predicting the flow behavior of jets used in industrial cleaning applications. Cavitation phenomenon is highly unstable [9]. Once the pressure around the bubble exceeds the pressure inside the bubble, the bubble will suddenly collapse [10]. In the very short time when the bubble collapses, hot spots appear in the cavitation bubble and the surrounding space. The maximum pressure and maximum temperature at the center of the cavitation bubble are about $80 \mathrm{MPa}$ and $6400 \mathrm{~K}$ [11], respectively. The rate of temperature change is as high as $107 \mathrm{~K} / \mathrm{s}$, accompanied by strong shock wave and micro-jet with a velocity of up to $400 \mathrm{~km} / \mathrm{h}$. Under such extreme conditions, free radical reactions, chemical bond breaking or high temperature cracking reactions occur in the organic matter in the fouling, so as to achieve the purpose of cavitation cleaning [12-13].

In this paper, the computational fluid dynamics software Fluent was used to simulate the hydrodynamic properties of water jet nozzle. The effects of structural parameters on the cavitation ability of the water jet nozzle was analyzed from the simulation. It is concluded that the larger vapor fraction and vapor fraction distribution region can be produced under the $25 \mathrm{MPa}$ inlet pressure. Through six groups of numerical simulation of different the length to diameter ratios, the cavitation quality of nozzles with different the length to diameter ratio of the nozzle throat section was obtained at a specific pressure inlet. It provides a theoretical basis for the design of nozzle structure.

\section{Establishment of nozzle structure model}

The standard $k-\varepsilon$ model may produce negatively normal stress when the time-averaged strain rate is very large. The standard $k-\varepsilon$ model may produce negative positive stress when the time-averaged strain rate is very large. Some restraint on normal stress is required. The realizable $k$ $-\varepsilon$ model is proposed to satisfy the physical laws of turbulence for fluid flow. In this paper, the flow field characteristics of water jet nozzles are studied, and the realizable $k-$ $\varepsilon$ model is selected to simulate the flow field inside the nozzles. However, one disadvantage of realizable $k-\varepsilon$ model is that it cannot provide natural turbulent viscosity when calculating rotational and static flow regions. This is because the effect of average curl is considered in the definition of turbulent viscosity in realizable $k-\varepsilon$ model. The nozzle structure model is shown in Fig. 1. When the fluid passes through the throat section, a certain pressure drop will be generated in the flow field to promote the generation of cavitation bubbles. The inlet diameter is $d 1$; The inlet contraction tube diameter is $D$; The diameter of the resonator is $\mathrm{d}$; The diameter of the throat section is $d 0$; The length of the resonator is $L 3$; The length of the throat section is $L 4$; The outlet diffusion angle $\alpha$ is $30^{\circ}$ [14]; The length of expansion section is $L 5$. The physical parameters of the nozzle are shown in Table 1. 


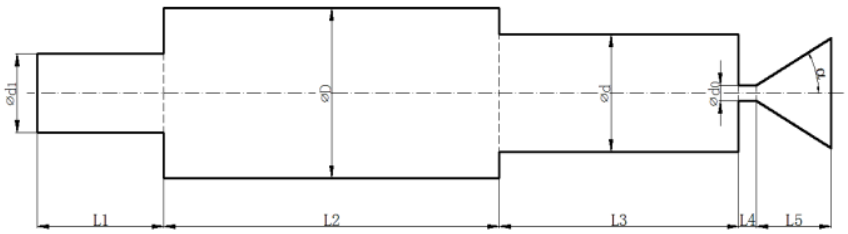

Fig. 1 Structural model of the nozzle

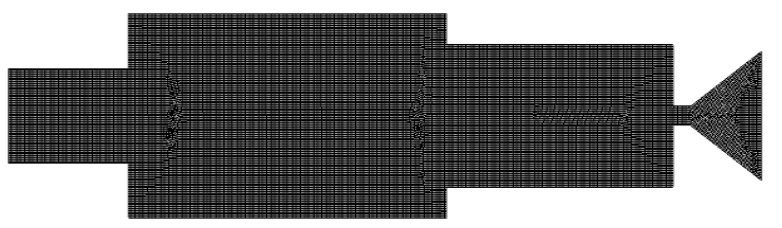

Fig. 2 Mesh model of the nozzle

Table 1

Physical parameters of the nozzle

\begin{tabular}{|c|c|c|c|c|c|c|c|c|c|c|}
\hline Projects & $L 1, \mathrm{~mm}$ & $L 2, \mathrm{~mm}$ & $L 3, \mathrm{~mm}$ & $L 4, \mathrm{~mm}$ & $L 5, \mathrm{~mm}$ & $D, \mathrm{~mm}$ & $d, \mathrm{~mm}$ & $d 0, \mathrm{~mm}$ & $d 1, \mathrm{~mm}$ & $\alpha,^{\circ}$ \\
\hline Parameters & 17 & 45 & 32 & 2.3 & 10 & 21.5 & 15 & 2 & 10 & 30 \\
\hline
\end{tabular}

Because the nozzle is a revolving body, the threedimensional model of the nozzle is simplified to a two-dimensional model [15]. The model is meshed by quadrilateral elements, and the flow field simulation of the nozzle is simulated by FLUENT software. In order to improve the calculation accuracy of the internal flow field, the mesh is refined [16]. The mesh model of the nozzle is shown in Fig. 2.

\section{Establishment of mathematical model}

After physical simplification of the model, the governing equations are established from the mathematical point of view, and are discretized and linearized to solve iteratively. Mixture model is a simplified multi-phase flow model, which can be used to simulate two-phase or multiphase flow with different velocities. It mainly solves the continuity equation, momentum equation, energy equation, volume fraction of the second phase and relative velocity equation of the Mixture phase. Therefore, the Mixture model is adopted.

\subsection{Multiphase flow model equation}

I) Continuous equation of Mixture model.

The continuity equation of the Mixture model is:

$$
\frac{\partial}{\partial t}\left(\rho_{m}\right)+\nabla \cdot\left(\rho_{m} \vec{v}_{m}\right)=\dot{m}
$$

where: $\dot{m}$ is to describe the mass transfer due to cavitation or user-defined mass sources; $\vec{v}_{m}$ is the average mass flow rate, which can be expressed by formula (2).

$$
\vec{v}_{m}=\frac{\sum_{k=1}^{n} \alpha_{k} \rho_{k} \vec{v}_{k}}{\rho_{m}}
$$

where: $\rho_{m}$ is the Mixture density, which can be expressed by formula (3).

$$
\rho_{m}=\sum_{k=1}^{n} \alpha_{k} \rho_{k}
$$

In formulas (2) and (3), $\alpha_{k}$ is the volume fraction of the first $k$ phase.

II) Momentum equation of Mixture model.

The momentum equation of the Mixture model can be obtained by summing the momentum equations of all phases [17]. It can be expressed as:

$$
\frac{\partial}{\partial t}\left(\rho_{m} \vec{v}_{m}\right)+\nabla \cdot\left(\rho_{m} \vec{v}_{m} \vec{v}_{m}\right)=-\nabla \rho_{m}+\rho_{m} \vec{g}+\vec{F}+\nabla \cdot\left[\mu_{m}\left(\nabla \vec{v}_{m}+\nabla \vec{v}_{m}^{T}\right)\right]+\nabla \cdot\left(\sum_{k=1}^{n} \alpha_{k} \rho_{k} \vec{v}_{d r, k} \vec{v}_{d r, k}\right), .
$$

where: $n$ is the phase number; $\vec{F}$ is the volume force; $\mu_{m}$ is the Mixture viscosity, which can be expressed by formula (5).

$$
\mu_{m}=\sum_{k=1}^{n} \alpha_{k} \mu_{k}
$$

where: $\vec{v}_{d r, k}$ is the drift velocity of the second phase $k$, which can be expressed by formula (6).

$$
\vec{v}_{d r, k}=\vec{v}_{k}-\vec{v}_{m}
$$

\subsection{Control equation}

The dynamic characteristics of a single cavitation bubble can be described by Rayleigh-Plesset equation [1]:

$$
R_{B} \frac{d^{2} R_{B}}{d t^{2}}+\frac{3}{2}\left(\frac{d R_{B}}{d t}\right)^{2}+\frac{2 S}{R_{B}}=\frac{p_{v}-p}{\rho_{l}},
$$

where: $R_{B}$ is the diameter of the cavitation bubble; $p$ is the confining pressure of the liquid; $\rho_{l}$ is the density of the liquid; $p_{v}$ is the saturated vapor pressure of the liquid; $S$ is the surface tension coefficient of the liquid. Regardless of the influence of the quadratic term and the surface tension in equation (7), the dynamic formula between the confining pressure of liquid and the radius of cavitation bubble is obtained.

$$
\frac{d R_{B}}{d t}=\sqrt{\frac{2\left|p_{v}-p\right|}{3 \rho_{l}}} .
$$

Formula (8) shows that the relationship between liquid confining pressure and mass change rate of a single cavitation bubble is as follows:

$$
\frac{d m_{B}}{d t}=4 \pi R_{B}^{2} \rho_{v} \sqrt{\frac{2\left|p_{v}-p\right|}{3 \rho_{l}}} .
$$


Assuming that there are $N_{B}$ cavitation bubbles in the unit volume, the expression of vapor volume fraction $\varphi_{v}$ is as follows:

$$
\varphi_{v}=V_{B} N_{B}=\frac{4}{3} \pi R_{B}^{3} N_{B}
$$

The mass conversion rate per unit volume can be calculated by substituting formula (10) into formula (9):

$$
T=\frac{d m_{B}}{d t}=\frac{3 \varphi_{v} \rho_{v}}{R_{B}} \sqrt{\frac{2\left|p_{v}-p\right|}{3 \rho_{l}}} .
$$

When $p<p_{v}$, the liquid will be cavitated. As cavitation nuclei grow into a cavitation bubble, the density of cavitation nuclei will decrease. Formula (10) can be expressed in formula (12):

$$
\varphi_{v}=\varphi_{n u c}\left(1-\varphi_{v}\right)
$$

where: $\varphi_{\text {nuc }}$ is the volume fraction of liquid cavitation nuclei.

The cavitation number [18-21] reflects the effect of absolute pressure on the generation of cavitation bubbles. The cavitation number is a special form of Euler number, which is the most important similarity criterion to describe the degree of cavitation development. The smaller the cavitation number, the larger the cavitation bubble inclusion region. It is defined as:

$$
\sigma=\frac{p_{\infty}-p_{v}}{\frac{1}{2} \rho_{l} v_{\infty}^{2}}
$$

where: $p_{\infty}$ is the inflow pressure of the liquid; $p_{v}$ is the saturated vapor pressure of the liquid; $p_{l}$ is the density of the liquid; $v_{\infty}$ is the inflow velocity of the liquid. It can be seen that the cavitation number $\sigma$ decreases with the increase of jet outlet velocity under a certain inflow pressure. If the number of primary cavitation bubbles in the jet is $\sigma_{i}$, then when $\sigma<\sigma_{i}$, cavitation bubbles will be generated in the jet.

\section{Setting of boundary conditions and simulation param- eters}

Mixture model is chosen for multiphase flow, and slip velocity between liquid phase and vapor phase is neglected. Because the size of the nozzle model is small, no large-scale bubbles will be generated, and the gravity effect can be neglected. Turbulence model chooses realizable $k-$ $\varepsilon$ model. The fluid property parameters are shown in Table 2.

Table 2

Physical properties of the two materials

\begin{tabular}{|c|c|c|}
\hline Properties & Water & Vapor \\
\hline Density, $\mathrm{kg} / \mathrm{m}^{3}$ & 998.2 & 0.5542 \\
\hline Viscosity, $\mathrm{kg} / \mathrm{m}^{1}$ & $1.003 \times 10^{-3}$ & $1.34 \times 10^{-5}$ \\
\hline
\end{tabular}

The main phase is water liquid. The second phase is water vapor. The conversion mode is cavitation, and the cavitation pressure is set as the saturated vapor pressure of water at 20 degrees Celsius, i.e. 2338.8 Pa. The inlet boundary is the pressure inlet, and the inlet pressure is setted as $15 \mathrm{MPa}$. The outlet boundary is the pressure outlet, and the outlet pressure is the standard atmospheric pressure, i.e. $101325 \mathrm{~Pa}$. Coupled is chosen as the solution method, and Zwart-Gerber-Belamri cavitation model is used for simulation analysis.

\section{Analysis of simulation results of nozzle flow field}

\subsection{Analysis of flow field inside nozzle}

Fig. 3 is a time-averaged pressure contour map inside the nozzle, from which it can be seen that the pressure inside the nozzle has almost no change from the inlet to the throat section. The pressure contour map of the nozzle throat section is shown in Fig. 4. There is a distinct pressure drop at the entrance of the nozzle throat section, forming a low pressure zone, which creates conditions for the cavitation inception of the fluid in the nozzle and the development of cavitation bubbles. When the minimum pressure in the flow field is less than the saturated vapor pressure at the local temperature of the liquid, cavitation will occur in the liquid.

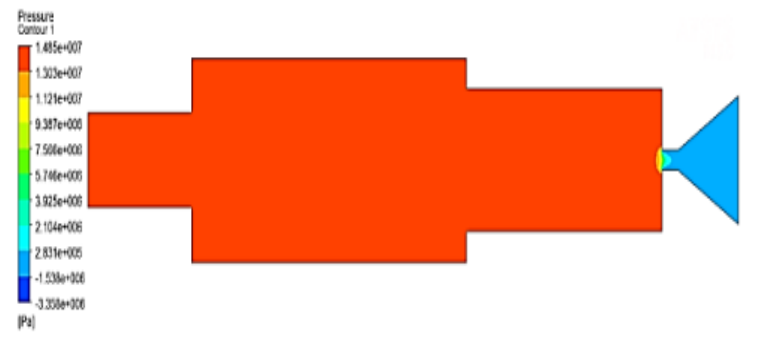

Fig. 3 Pressure contour map of the nozzle

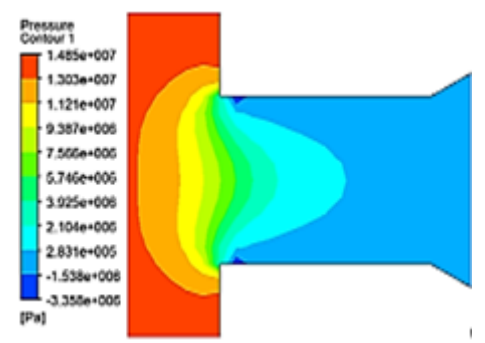

Fig. 4 Pressure cloud map of the throat section of the nozzle

Fig. 5 is a time-averaged velocity contour map inside the nozzle, from which it can be seen that the flow velocity inside the nozzle does not increase significantly in the inlet contraction tube and the resonator. The velocity increases as the fluid flows through the throat section of the nozzle where the fluid velocity reaches a maximum. When the liquid leaves the nozzle outlet at a high speed, a Local reflux zone is formed in the nozzle expansion section. As shown in Fig. 6, the reflux causes the vapor fraction distribution region of the expanded section to gradually spread along the expansion section wall.

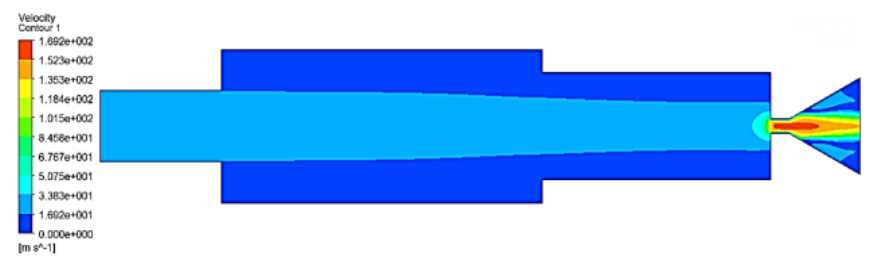

Fig. 5 Velocity contour map of the nozzle 


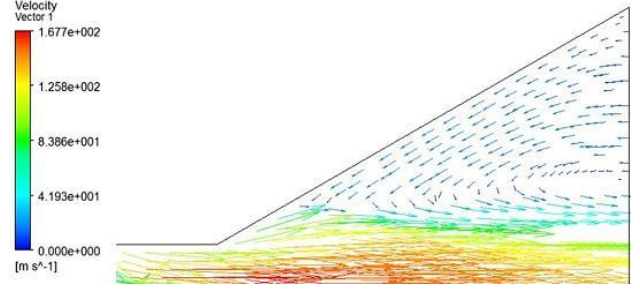

Fig. 6 Schematic diagram of flow in the nozzle expansion section

In Fig. 7, the time-averaged vapor volume distribution of the nozzle shows that cavitation occurs when the fluid passes through the nozzle throat section. Cavitation bubbles occur near the wall of the nozzle throat section, and the maximum vapor fraction occurs at the beginning of the expansion section. When cavitation occurs in the flow field, strong disturbance and turbulent kinetic energy increase sharply, as shown in Fig. 8. The variation range of turbulent kinetic energy reflects the strength of cavitation. The generation, growth and collapse of cavitation bubbles will cause disturbance of flow field, and the turbulent kinetic energy will increase with the increase of fluctuating pressure. The maximum turbulent kinetic energy occurs on both sides of the nozzle outlet axis, not on the nozzle outlet axis.

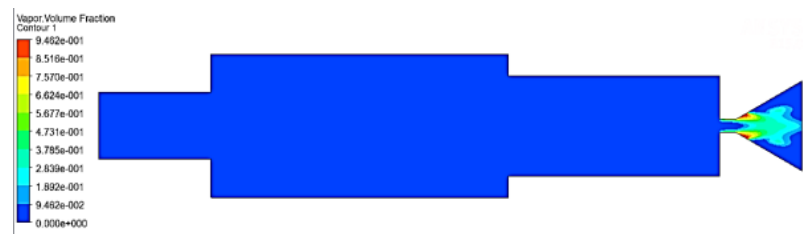

Fig. 7 Vapor phase volume fraction diagram of the nozzle

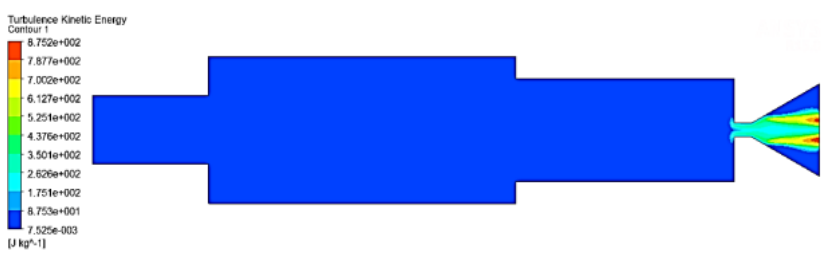

Fig. 8 Turbulent kinetic energy distribution diagram of the nozzle

\subsection{Cavitation vapor fraction distribution under different conditions}

In order to study the change of flow field in nozzle under different pressure inlets, six groups of parameters, 5 , $10,15,20,25$ and $30 \mathrm{MPa}$, were used to simulate the flow field. The results show that, as shown in Fig. 9, the pressure of six groups of pressure inlet parameters will drop suddenly in the nozzle throat section, and the minimum pressure is almost the same, all of them will fall below the saturated vapor pressure. The nozzle exit velocity increases linearly with the increase of pressure inlet, as shown in Fig. 10. When the fluid passes through the throat section of the nozzle, the flow rate rises sharply and then decreases slightly. This is because when the fluid passes through the throat section of the nozzle, cavitation bubbles begin to form, and then the cavitation bubbles grow, and a large number of cavitation bubbles form a cavitation cloud. The core region has highly concentrated kinetic energy, so the length of the core region is expected to increase with the increase of jet pressure. When the cavitation cloud expands, the flow of fluid will be blocked, resulting in a small decrease in the nozzle exit velocity.

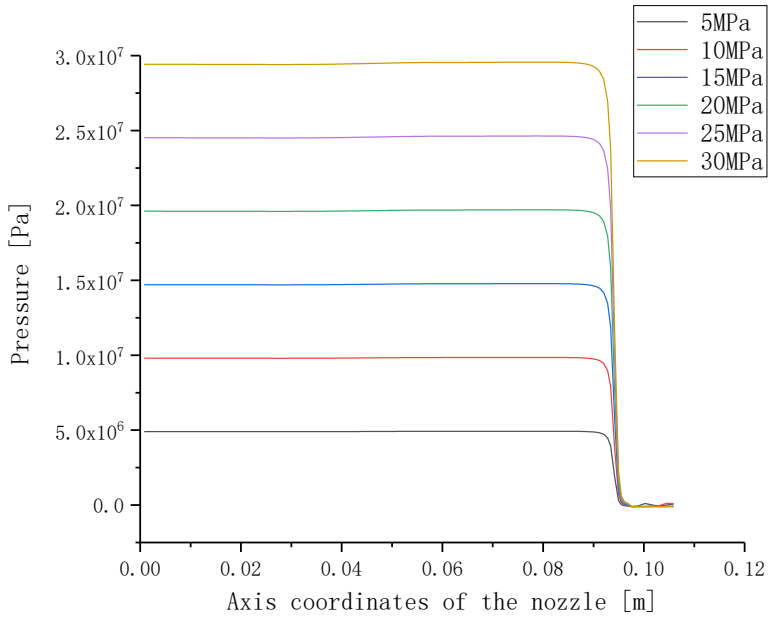

Fig. 9 Pressure curves distribution of the nozzle axis

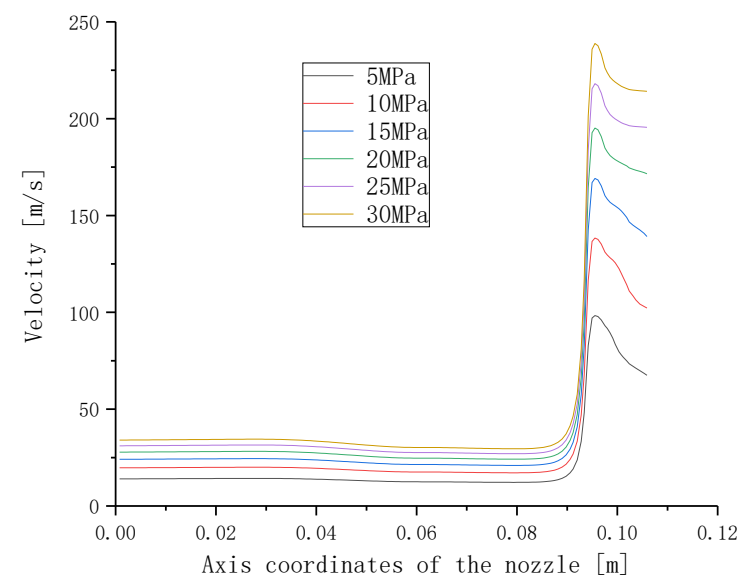

Fig. 10 Velocity curve distribution of the nozzle axis

Fig. 11 shows the time-averaged vapor phase volume fraction distribution of nozzle expansion section under different pressure inlet. It can be seen from the diagram that the vapor fraction begins to diffuse along the wall from the starting point of the nozzle throat section and the starting point of the expansion section. Fig. 12 shows vapor fraction curves distribution of nozzle expansion section wall under different pressure inlet. Combining with Figs. 11 and 12, when the inlet pressure is $5 \mathrm{MPa}$, the maximum vapor fraction is 0.84 , which occurs at the starting point of the nozzle expansion section, and the vapor fraction distribution region is small. As the pressure inlet increases gradually, the maximum vapor fraction increases, and the distribution region of vapor fraction increases accordingly. The maximum vapor fraction region increases gradually along the diffusion wall, especially when the pressure inlet increases to $25 \mathrm{MPa}$, the vapor fraction reaches 0.9924 . It can be seen that the intensity of cavitation is very intense, and the distribution region of vapor fraction also increases sharply, almost full of the whole expansion section of the nozzle. The pressure inlet was increased to $30 \mathrm{MPa}$, and the vapor fraction value and the vapor fraction distribution region were not substantially increased. This shows that there is an optimum value for the influence of the pressure inlet on the distribution region and the vapor fraction of the cavitation bubble at the expansion section. The reason for this phenomenon is related to the number of cavitation nuclei. Because there is a 
reflux in the expansion section of the nozzle, the reflux of the cavitation nuclei in other region gather on the wall of the expansion section. The greater the number of cavitation nuclei, the greater the number of cavitation bubbles, and the more cavitation.

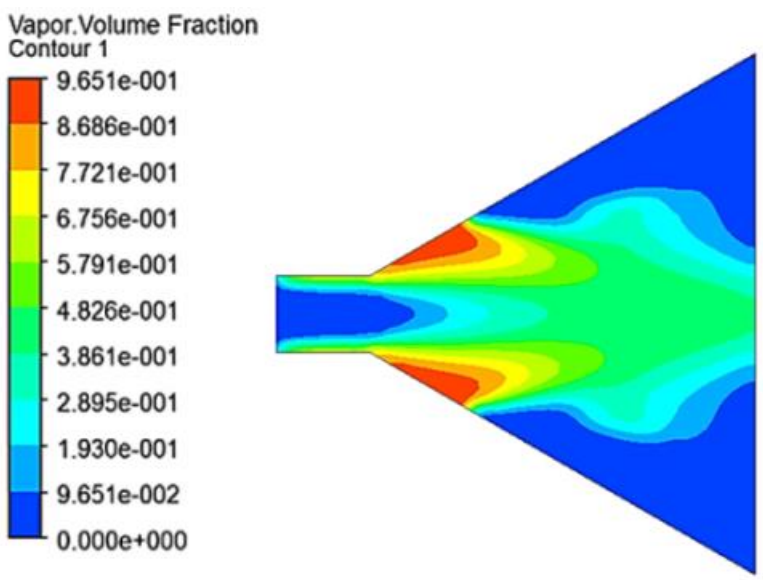

d) $20 \mathrm{MPa}$ pressure inlet

a) $5 \mathrm{MPa}$ pressure inlet

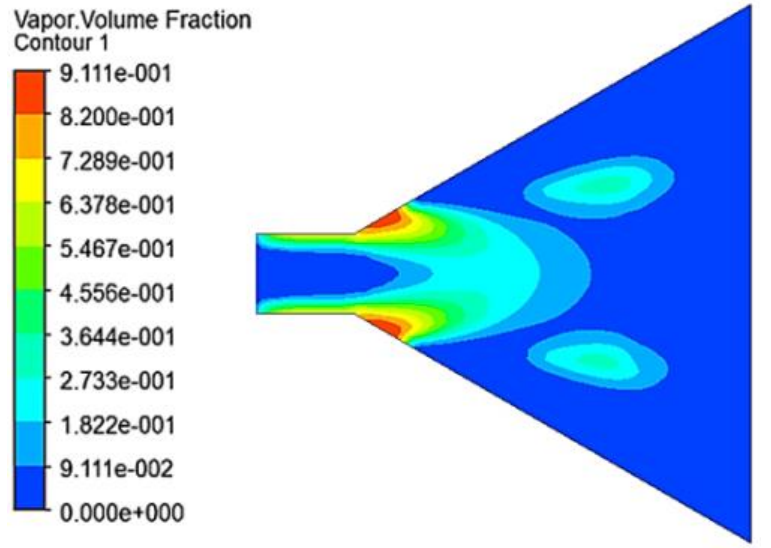

b) $10 \mathrm{MPa}$ pressure inlet

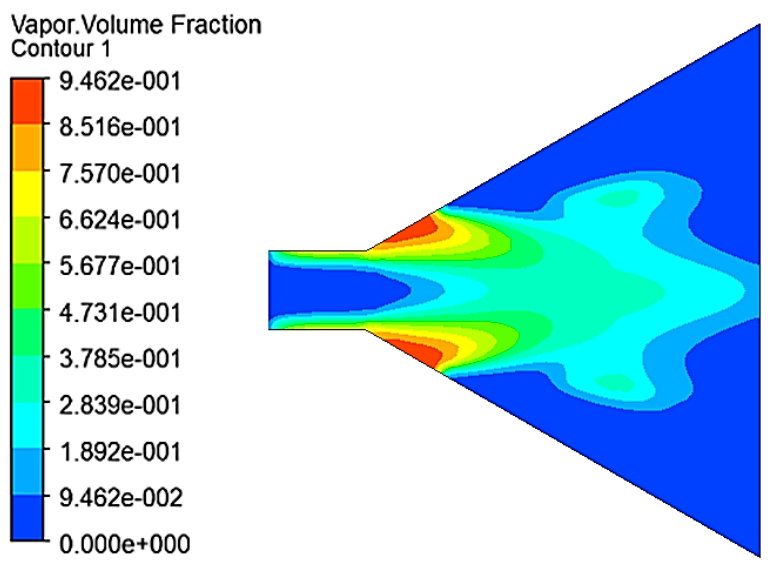

c) $15 \mathrm{MPa}$ pressure inlet

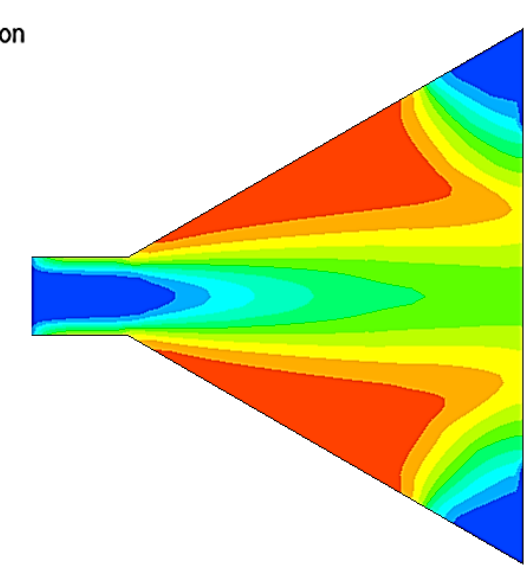

e) $25 \mathrm{MPa}$ pressure inlet

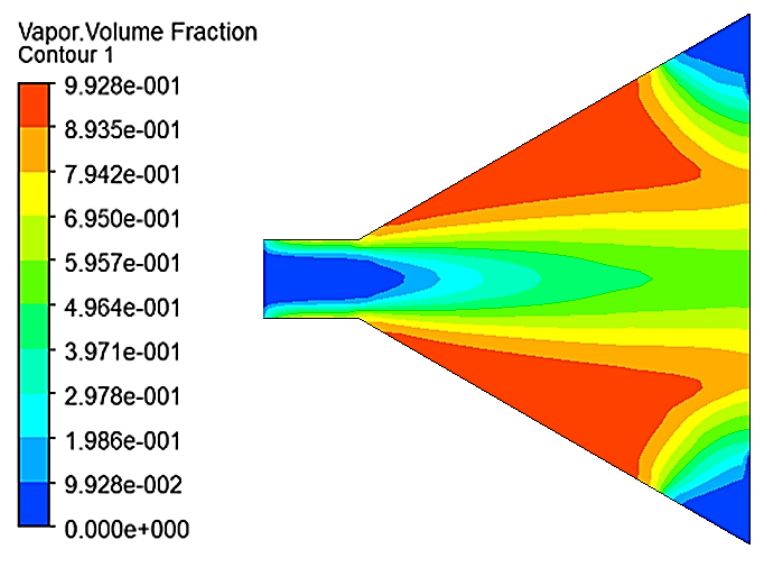

e) $30 \mathrm{MPa}$ pressure inlet

Fig. 11 Vapor phase volume fraction distribution of nozzle expansion section under different pressure inlet

In order to further study the influence of different the length to diameter ratio $(L 4 / d 0)$ on the vapor fraction at the nozzle throat section, six groups of parameters, namely, the length to diameter ratio of $0.1,0.5,1,1.5,2$ and 2.5 , were numerically simulated under the pressure inlet of $25 \mathrm{MPa}$. As a result, as shown in Fig. 13, when the length to diameter ratio is 0 , the number of cavitation bubbles in the expansion section is small, the vapor fraction value is low, and the vapor fraction distribution region is also small. When the length to diameter ratio is 0.5 , the vapor fraction value increases, but the vapor fraction distribution region is still small. When the length to diameter ratio is 1 , the vapor fraction of the expansion section increases, and the vapor fraction distribution region also increases. When the length to 
diameter ratio is 1.5 , the vapor fraction of the expansion section rises slightly, but the vapor fraction distribution region suddenly increases, almost filling the entire expansion section of the nozzle. It is because the change of the length to diameter ratio increases the water flow disturbance, affects the turbulent kinetic energy, and then increases the vapor fraction distribution region. When the length to diameter ratio is 2 , the vapor fraction value does not increase, and the vapor fraction distribution region only slightly expanded. When the length to diameter ratio is 2.5 , the vapor fraction value and the vapor fraction distribution region of the expansion section are substantially no longer increased when the length to diameter ratio is 2 . Therefore, different length to diameter ratio has a great influence on the cavitation quality.

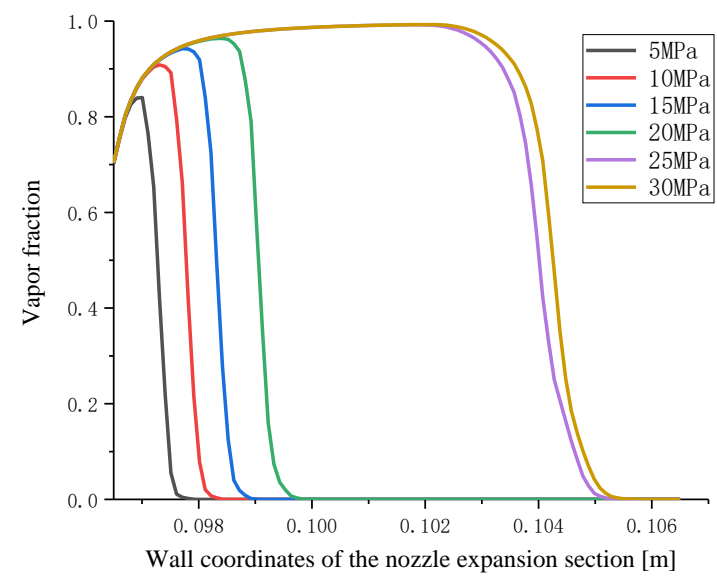

Fig. 12 Vapor fraction distribution curve of nozzle expansion section wall under different pressure inlet

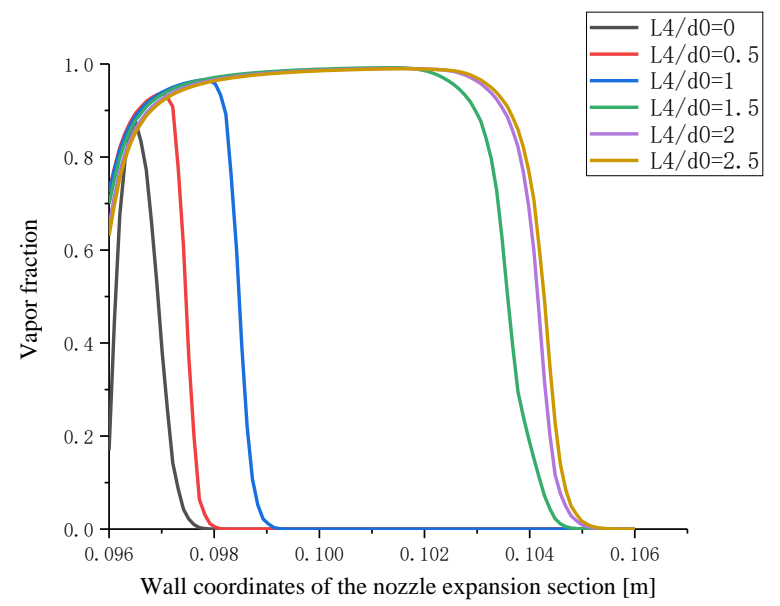

Fig. 13 Vapor fraction distribution curve of nozzle expansion section wall under different length to diameter ratio

\section{Conclusions}

1. Cavitation occurs first in the throat section of the nozzle and the starting point of the expansion section, and the high vapor fraction region occurs in the wall of the nozzle expansion section. In addition, the reflux of the fluid causes the vapor fraction distribution region to gradually spread along the wall of the expansion section with the pressure inlet increases.

2. With the gradual increase of the pressure inlet, the vapor fraction and the vapor fraction distribution region of the nozzle expansion section are also gradually increased.
Especially when the pressure inlet is $25 \mathrm{MPa}$, the vapor fraction and the vapor fraction distribution region in the expansion section are basically up to the maximum, and the cavitation quality is better.

3. The length to diameter ratio has little effect on the vapor fraction of the expansion section, but the length to diameter ratio has a greater influence on the vapor fraction distribution region. When the length to diameter ratio is 2 , the vapor fraction distribution region reaches the maximum.

Reference [4] only qualitatively illustrates that increasing the length-diameter ratio of the nozzle throat is equivalent to increasing the low pressure area. In this paper, we quantitatively study the distribution of the vapor fraction distribution curve of nozzle expansion section wall under different length to diameter ratio. Because of the limited conditions, this paper only simulates the flow field of the nozzle. In the process of simulation analysis, in order to simplify the simulation analysis model, the 3D model of the nozzle is simplified to $2 \mathrm{D}$ model. In the future research, the simulation results should be further analyzed combined with experiments.

\section{Acknowledgments}

The project was supported by a key research project of Higher Education of Henan Province (20A460019).

\section{References}

1. Zhao, Ding-xuan; Wang, Qian; Du, Miao-miao. 2016. Fluent-based numerical simulation of cavitation behavior in the angle nozzle, Journal of Northeastern University (Natural Science) 37(9): 1283-1287. http://dx.doi.org//10.3969/j.issn.10053026.2016.09.015.

2. Hong, Feng; Gao, Zhenjun; Yuan. Jianping. 2018. Improved cavitation model based on rayleigh-plesset equation and its application, Transactions of the Chinese Society for Agricultural Machinery 49(2): 126-132. http://dx.doi.org//10.6041/j.issn.10001298.2018.02.017.

3. Lu, Yi-yu; Wang, Xiao-chuan; Kang, Yong; et al. 2009. Numerical simulation for cavitation water jet flow field through convergent-divergent nozzle, Journal of China University of Petroleum 33(6): 57-60. http://dx.doi.org//1673-5005(2009)06-0057-04.

4. Hu, Kun; AIi, Zhijiu; Fu, Biwei; et al. 2013. Numerical simulation of flow field for organ-pipe cavitation jet nozzle, Science \& Technology Review 31(21): 44-47. http://dx.doi.org//10.3981/j.issn.10007857.2013.21.007.

5. Liu, Wenchuan; Kang, Yong; Zhang, Mingxing; et al. 2017. Self-sustained oscillation and cavitation characteristics of a jet in a Helmholtz resonator, International Journal of Heat and Fluid Flow 68: 158-172.

http://dx.doi.org//10.1016/j.ijheatfluidflow.2017.10.004.

6. Wenchuan, Liu; Kang, Yong; Mingxing, Zhang; et al. 2018. Experimental and theoretical analysis on chamber pressure of a self-resonating cavitation waterjet, Ocean Engineering 151: 33-45.

http://dx.doi.org//10.1016/j.oceaneng.2018.01.019.

7. Huang, B.; Wang, G.; Zhang, B.; et al. 2010. Evaluation and application of filter based turbulence model for 
computations of cloud cavitating flows, Journal of Mechanical Engineering 46(8):147-153. http://dx.doi.org//10.3901/JME.2010.08.147.

8. Anirban, Guha; Ronald, M. Barron; Ram, Balachandar. 2011. An experimental and numerical study of water jet cleaning process, Journal of Materials Processing Technology 211(4): 610-618. http://dx.doi.org//10.1016/j.jmatprotec.2010.11.017.

9. Hong, Liu; Chang, Cai; Xi, Xi, et al. 2018. A novel model for the bubble growth in the cavitation region of an injector nozzle, International Journal of Heat and Mass Transfer 119: 128-138. http://dx.doi.org//10.1016/j.ijheatmasstransfer.2017.11. 101

10. Peters, A.; Sagar, H.; Lantermann, U., et al. 2015. Numerical modeling and prediction of cavitation erosion, Wear 338-339:189-201. http://dx.doi.org//10.1016/j.wear.2015.06.009.

11. Fujikawa, S; Akamatsu, T. 1980. Effects of the nonequilibrium condensation of vapour on the pressure wave produced by the collapse of a bubble in a liquid, Fluid Mesh 97: 481-512. http://dx.doi.org//10.1017/S0022112080002662.

12. Peters, A.; Lantermann, U.; Ould el Moctar. 2018. Numerical prediction of cavitation erosion on a ship propeller in model- and full-scale, Wear 408-409. http://dx.doi.org//10.1016/j.wear.2018.04.012.

13. Verhaagen, B.; Fernández Rivas, D. 2016. Measuring cavitation and its cleaning effect, Ultrasonics Sonochemistry 29: 619-628. http://dx.doi.org//10.1016/j.ultsonch.2015.03.009.

14. Zhang, F.; Tang, Ch.; Yang, L.; et al. 2004. Investigation of Cavitating nozzle with converging-diverging exit, Fluid Machinery 32(1): 25-28. http://dx.doi.org//1005-0329(2004)01-0025-04.

15. Liang, Y. 2016. Research and Development of Cavitation Jet Device. Hangzhou: Zhejiang University of Technology, 2016.

16. Kewen, Peng; Shouceng, Tian; Gensheng, Li; et al. 2018. Mapping cavitation impact field in a submerged cavitating jet, Wear 396-397: 22-33. http://dx.doi.org//10.1016/j.wear.2017.11.006.

17. Jiang, Fan; Huang, Peng. 2008. Fluent advanced application and example analysis. BeiJing: Tsinghua University Press. 147-148 p.

18. Wang, X. 2009. Cavitating and supercavitating flows theory and applications, Beijing: National Defense Industry Press. 27-53 p.

19. Wang, X.; Jiao, L.; Wang, L. 2005. Numerical simulation of self-exicited oscillation Pulsed jet and analysis of
Parameters' influence, Journal of Zhejiang University (Engineering Science) 39(9): 1450-1454. http://dx.doi.org//1008-973X(2005)09-1450-05.

20. Ma, B.; Gao, Y.; Liu, Y.; et al. 2017. Establishment and experimental validation of nozzle cavitation model, Transactions of the Chinese Society for Agricultural Machinery 48(5): 377-384.

http://dx.doi.org//10.6041/j.issn.10001298.2017.05.048.

21. Sou, A. 2009. Liquid jet deformation induced by cavitation in nozzles of various shapes, Japanese Journal of Applied Physics 2(2): 694-702. http://dx.doi.org//10.4271/2009-24-0157.

\section{Y. Q. Su, J. F. Shi, Y. H. Wang}

\section{NUMERICAL SIMULATION OF CAVITATION OF WATER JET NOZZLE BASED ON REALIZABLE $k-\varepsilon$ MODEL}

S u m m a r y

In order to study the cavitation characteristics of water jet nozzles, a realizable $k-\varepsilon$ model was selected to simulate the flow field inside the nozzle at the inlet pressure of $15 \mathrm{MPa}$. The pressure at the starting point of the nozzle throat section dropped to the minimum, and the liquid velocity reached the maximum. From the vapor volume distribution map of the nozzle, it can be seen that the vapor fraction is the largest on the wall of the expansion section, and a local reflux is formed in the expansion section, which results in the gradual diffusion of the vapor fraction distribution along the wall of the expansion section. In addition, the influence of nozzle inlet pressure on the vapor fraction and vapor fraction distribution region in the expansion section is analyzed. The results show that the larger vapor fraction and vapor fraction distribution region can be produced under the $25 \mathrm{MPa}$ inlet pressure. At the pressure inlet of $25 \mathrm{MPa}$, six groups of numerical simulations were carried out with different the length to diameter ratio of the nozzle throat section $(L 4 / d 0)$. The results show that when $L 4 / d 0$ is 2 , it is more conducive to the formation of cavitation and the quality of cavitation is better.

Keywords: cavitation jet, nozzle, flow field, numerical analysis, vapor fraction.

Received March 02, 2021 Accepted February 04, 2022 